\title{
Editorial
}

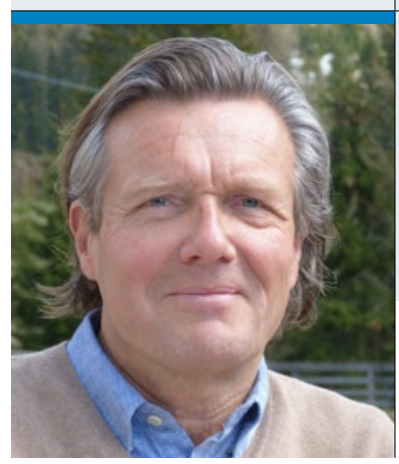

„Für mehr Transparenz und weniger Bürokratie wäre es sinnvoll, alle Krankenversicherungen der BVA zu unterstellen."

Prof. Dr. med Gerhard Grevers

Chefredaktion

\section{Mauscheleien und kein Ende}

D ie Gaunereien der Versicherer im Gesundheitswesen, - auch so eine „never ending story". In Ausgabe 6/2016 der HNO-Nachrichten hatte ich ja bereits über die zweifelhaften Praktiken der gesetzlichen Krankenkassen berichtet. Jetzt legt die WirtschaftsWoche nach: Auf der Titelseite von Heft 10/17 heißt es in großen Lettern: „Kasse voll, Patient tot, wie gesetzliche Krankenkassen tricksen, täuschen und Patienten schikanieren". Ich hatte seinerzeit schon das beliebte "upcoding“ erwähnt, ein Euphemismus für die vor allem in Krankenhäusern beliebte Diagnosefälschung. In Kassenkreisen wird das auch unverblümt artikuliert. Jens Baas von der Techniker Krankenkasse wird dazu freimütig mit den Worten zitiert: „Die Kassen bezahlen zum Beispiel Prämien von $10 €$ je Fall für Ärzte, die Patienten auf dem Papier kränker machen". Bananenrepublik Deutschland halt. Dass "Abrechnungsoptimierung“, wie es so schön heißt, illegal ist, darauf haben Bundesgesundheitsministerium und Bundesversicherungsanstalt zwar längst hingewiesen, aber wen interessiert das hierzulande schon. Die AOK Rheinland hat man jetzt immerhin wegen derartiger Manipulationen am Haken; Konsequenz: 7 Millionen $€$ Strafe - aus Versichertengeldern. Das ist natürlich gar nichts, verglichen mit den 200 Milliarden, welche die Gesetzlichen jedes Jahr von ihrer Klientel abgreifen. Wenn's mal teurer werden könnte bei den Strafzahlungen, wählt man auch gerne mal den kleinen Dienstweg über willfährige Volksvertreter: 2016 kam es zum Streit um Zahlungen aus dem Gesundheitsfond für Auslandsversicherte. Die Kassen hatten mehr Geld erhalten als die Versicherten kosteten. Rechtzeitige Intervention aus der Politik war erfolgreich: ein Gesetzesentwurf, der die AOK Rheinland/Hamburg Millionen an Rückzahlungen gekostet hätte, wurde einfach vertagt. Das bringt selbst altgediente Parteisoldaten in Rage. Franz Knieps, als Ex-Abteilungsleiter unter Ulla Schmidt mit verantwortlich für das kranke Gesundheitswesen in Deutschland, trat nach mehr als 30 Jahren Parteizugehörigkeit aus der SPD aus. Gründe unter anderem: die unerträgliche Lobbymacht der gesetzlichen Kassen und hier vor allem der AOK, die „in manchen Regionen wie ein Teil der Landesregierung wirkt". Im Unterschied zu den übrigen Krankenkassen, die von der Bundesversicherungsanstalt (BVA) kontrolliert werden, unterliegt die AOK nämlich primär der Aufsicht der Länder. Sinnvoller wäre es, alle Versicherungen der BVA zu unterstellen. Das würde nicht nur den unnötigen bürokratischen Aufwand verringern, sondern auch mehr Transparenz schaffen; freiwerdende Ressourcen kämen dann endlich denen zugute, die das Ganze bezahlen: den Versicherten.

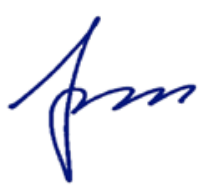

